\title{
No Changes in Functional Connectivity After Dimethyl Fumarate Treatment in Multiple Sclerosis
}

\author{
Claudia Piervincenzi · Emilia Sbardella · Marta Altieri · \\ Antonio Ianniello · Patrizia Pantano (D) - Carlo Pozzilli • \\ Nikolaos Petsas
}

Received: October 23, 2021 / Accepted: January 19, 2022 / Published online: February 4, 2022

(C) The Author(s) 2022

\section{ABSTRACT}

Introduction: Despite the increased availability of disease-modifying therapies (DMTs) for treating relapsing-remitting multiple sclerosis (RR-MS), only a few studies have evaluated DMT-associated brain functional changes.

Methods: We investigated whether significant resting-state functional connectivity (FC) changes occurred in RR-MS patients after 6 and 12 months of dimethyl fumarate (DMF) treatment using both a seed-based and data-driven approach.

Results: Thirty patients were followed up after 6 months of therapy, and 27 of them reached a

C. Piervincenzi · M. Altieri · A. Ianniello ·

P. Pantano $(\varangle)$. C. Pozzilli

Department of Human Neurosciences, Sapienza

University of Rome, Rome, Italy

e-mail: patrizia.pantano@uniroma1.it

P. Pantano $\cdot$ N. Petsas

IRCCS NEUROMED, Pozzilli, IS, Italy

A. Ianniello · C. Pozzilli

Multiple Sclerosis Centre, Sant'Andrea Hospital, Rome, Italy

E. Sbardella

Department of Experimental Medicine, Sapienza

University of Rome, Rome, Italy 12-month follow-up. Three patients at baseline and only one after 12 months showed gadolinium-enhancing lesions. We did not find any significant FC changes after therapy at either time point. After 12 months of DMF, we observed relatively modest brain volume loss and a significant improvement in Paced Auditory Serial Addition Test $3 \mathrm{~s}$ and 25-Foot Walk Test scores.

Conclusion: The absence of FC changes could be due to the low degree of baseline inflammation in our patients, though we cannot exclude that more time may be required to observe such changes. No FC changes may reflect a beneficial effect of DMF therapy, as supported by conventional MRI findings and clinical improvement.

Keywords: Multiple sclerosis (MS); Restingstate functional MRI; Functional connectivity; Dimethyl fumarate (DMF); Disease-modifying therapy (DMT) 


\section{Key Summary Points}

We investigated whether significant functional connectivity changes occur in relapsing-remitting multiple sclerosis patients after 6 and 12 months of dimethyl fumarate treatment

No significant functional connectivity changes were found after 6 or 12 months of dimethyl fumarate treatment

After 12 months of therapy, relatively modest brain volume loss was found, along with a significant improvement in Paced Auditory Serial Addition Test $3 \mathrm{~s}$ and 25-Foot Walk Test scores

The lack of functional connectivity changes may be due to the low degree of baseline inflammation in our patient cohort, suggesting that more time may be required to observe such changes

The lack of functional connectivity changes, along with modest volume loss and clinical improvement, may reflect a positive phenomenon (i.e., stability)

\section{INTRODUCTION}

Despite the increased availability of diseasemodifying therapies (DMTs) for treating relapsing-remitting multiple sclerosis (RR-MS), only a few studies have evaluated DMT-associated brain functional changes [1]. In two recent studies, we investigated resting-state functional connectivity (FC) changes after initial treatment with fingolimod in RR-MS [2, 3]. In the first study, we found a significant decrease in FC between the primary motor cortex and posterior cortical areas, which correlated with a significant improvement in information processing speed [2]. In the second study, we used a datadriven approach (independent component analysis, ICA) to investigate large-scale network DMT-related FC changes and observed that only patients who reached NEDA (no evidence of disease activity) status [4] showed significant within- and between-network FC changes after fingolimod treatment. Moreover, NEDA status was predicted by higher within-network FC at baseline [3].

Another first-line treatment for RR-MS is dimethyl fumarate (DMF), a widely used oral DMT licensed in 2013 on the basis of two positive phase III studies $[5,6]$, which showed that DMF significantly reduced the proportion of patients who had a relapse over 2 years, as well as the annualized relapse rate, the rate of disability progression, and the number of lesions on MRI. DMF suppresses inflammatory response and protects against nerve cell death [7].

We hypothesized that beneficial effects of DMF are associated with reduced FC, as we previously demonstrated in patients treated with fingolimod $[2,3]$. Therefore, the present study aimed to investigate (1) whether FC changes occur after 6 and/or 12 months of DMF therapy and (2) whether FC changes, if present, are comparable to those observed after fingolimod therapy $[2,3]$.

\section{METHODS}

\section{Participants}

Thirty-nine RR-MS patients were recruited and underwent MRI scanning and clinical testing at Policlinico Umberto I, Sapienza University of Rome, Italy.

Inclusion criteria were: age range 18-65 years; diagnosis of MS according to the revised McDonald 2010 criteria [8]; relapsingremitting form; eligibility to be treated with DMF according to European Medicines Agency indications; right-handedness.

Exclusion criteria were: concomitant serious systemic disease, steroid administration and relapse within 2 months of study entry, prior history of other neurological or psychiatric disorders, and/or contraindications to MRI.

MRI and clinical/neuropsychological data were acquired 1-2 days prior to starting DMF (therapy start-Tst), after 6 months (T6m), and after 12 months (T12m). 
At each time point, the following clinical/ neuropsychological measures were collected: Expanded Disability Status Scale (EDSS) score and MS Functional Composite (MSFC) score with its sub-scores [9-Hole Peg Test (9-HPT), 25-Foot Walk Test (25-FWT), and Paced Auditory Serial Addition Test (PASAT) 3 and $2 \mathrm{~s}$ ] and Symbol-Digit Modalities Test (SDMT) score. At the follow-up examination, disability progression with respect to baseline evaluation was defined as a 1.5-point increase for patients with a baseline EDSS score of 0, a 1-point increase for patients with EDSS scores from 1.0 to 5.0, and a 0.5 -point increase for patients with EDSS scores equal to or higher than 5.5 [9].

\section{Ethics Statement}

This study was performed in accordance with the ethical code of the ethics committee of Azienda Policlinico Umberto I, Sapienza University of Rome (Ref. 2984/12.12.2013), and the Declaration of Helsinki. After approval from the ethics committee, written informed consent was obtained from all subjects.

\section{MRI Acquisition}

Images were acquired with a 3-T scanner (Siemens Magnetom Verio) and a 12-channel head coil designed for parallel imaging (GRAPPA). Participants were advised to avoid consuming psychoactive substances, such as tea or coffee, within $2 \mathrm{~h}$ prior to MRI scans. The following sequences were acquired:

- Blood oxygen level-dependent (BOLD) single-shot echo-planar imaging [repetition time $(\mathrm{TR})=3000 \mathrm{~ms}$, echo time $(\mathrm{TE})=30 \mathrm{~ms}$, flip angle $=89^{\circ}$, field of view $($ FOV $)=192 \mathrm{~mm}, 64 \times 64$ matrix, 50 contiguous axial slices $3 \mathrm{~mm}$ thick, 140 volumes, voxel size $=3 \mathrm{~mm}^{3}$, acquisition time $=7 \mathrm{~min} 11 \mathrm{~s}]$, with all patients instructed to close their eyes and stay awake during the resting-state fMRI acquisitions;

- High-resolution 3D T1-weighted (T1-3D) MPRAGE sequence [TR $=1900 \mathrm{~ms}$, $\mathrm{TE}=2.93 \mathrm{~ms}$, inversion time $(\mathrm{TI})=900 \mathrm{~ms}$, flip angle $=9^{\circ}, \mathrm{FOV}=260 \mathrm{~mm}$, matrix $=256$ $\times 256,176$ sagittal slices $1 \mathrm{~mm}$ thick, no gap];

- Dual turbo spin-echo, proton density (PD) and T2-weighted images $(\mathrm{TR}=3320 \mathrm{~ms}$, $\mathrm{TE} 1=10 \mathrm{~ms}, \mathrm{TE} 2=103 \mathrm{~ms}, \mathrm{FOV}=220 \mathrm{~mm}$, matrix $=384 \times 384,25$ axial slices $4 \mathrm{~mm}$ thick, 30\% gap);

- T1-weighted spin-echo sequence acquisition after administration of a gadolinium-based contrast agent $(\mathrm{TR}=550 \mathrm{~ms}, \mathrm{TE}=9.8 \mathrm{~ms}$, $\mathrm{FOV}=240 \mathrm{~mm}, \quad$ matrix $=320 \times 320, \quad 25$ axial slices $4 \mathrm{~mm}$ thick, 30\% gap).

\section{MRI Analysis}

\section{Lesion and Brain Volume}

Lesion volume was calculated on PD images using Jim 5.0 software (Xinapse System, Leicester, UK; http://www.xinapse.com).

Measures of global brain volume and gray matter (GM) volume at baseline (Tst) were obtained from lesion-filled T1-3D brain images using SIENAX, while measures of percentage brain volume change (PBVC) between Tst and $\mathrm{T} 6 \mathrm{~m}$ and between Tst and $\mathrm{T} 12 \mathrm{~m}$ were obtained using SIENA, part of FMRIB's Software Library (FSL) (https://fsl.fmrib.ox.ac.uk/fsl/fslwiki).

\section{Functional Connectivity}

Functional MRI data were preprocessed using FSL, v5.0.9. Single-subject preprocessing was performed using FEAT (FMRI Expert Analysis Tool) and included motion correction, nonbrain substance removal, spatial smoothing, and high-pass filtering. At this stage, we also carried out both linear and non-linear registration to obtain functional-to-standard transformation matrices. Four-dimensional GM maps were obtained with the feat_gm_prepare tool and used as voxel-wise nuisance variables in subsequent statistical analyses. Preprocessing details are reported in $[2,3]$. We performed a seed-towhole-brain interregional regression analysis placing the seed on the right hand cortical representation (left primary motor cortex) [2] and an ICA analysis using MELODIC tool (Multivariate Exploratory Linear Optimized Decomposition into Independent Components) 
[10] followed by dual-regression analysis [11]. Resting-state networks (RSNs) of interest were identified via spatial correlation coefficients using RSNs generated by Smith et al. [12] and Yeo et al. [13] as templates and then verified by expert visual inspection (CPi, NP, PP).

Subject-level difference maps ( $\triangle \mathrm{FC}$ maps) between $\mathrm{T} 6 \mathrm{~m}$ and Tst and between $\mathrm{T} 12 \mathrm{~m}$ and Tst were obtained for primary motor cortex FC maps and for each RSN and used for further group-level voxel-wise analyses.

\section{Statistical Analyses}

Statistical analyses of demographic, clinical, radiological, and neuropsychological parameters were performed using SPSS statistics software (version 22.0). Longitudinal changes in clinical, neuropsychological, and radiological parameters were estimated using Wilcoxon signed-rank test $(p<0.05$ for null hypothesis rejection).

Primary motor cortex FC changes were investigated performing one-sample $t$-tests on $\Delta$ FC maps using FEAT. Age, sex, and GM maps were included as nuisance variables. $Z$ statistic images were thresholded non-parametrically using clusters determined by $Z>2.3$ and a (corrected) cluster significance threshold of $p=0.05$ [14].

To investigate within-network FC changes, one-sample $t$-tests were performed on $\Delta \mathrm{FC}$ maps, with age, sex, and GM maps as nuisance variables. Voxel-wise statistical analyses were performed with permutation-based non-parametric statistics using FSL Randomise permutation-based program with 5000 permutations [15]. Clusters were determined by using threshold-free cluster enhancement (TFCE) [16] and a family-wise error (FWE)-corrected cluster significance threshold of $p<0.05$.

Between-network FC differences were investigated using FSLNets toolbox (http://fsl.fmrib. ox.ac.uk/fsl/fslwiki/FSLNets). After normalization of the extracted time courses of all RSNs identified in each subject, the time courses of artifactual components and components of no interest were regressed out of the individual data. Subject-wise correlation matrices of both full and partial correlations of all remaining RSN time courses were then created. Betweensubject testing was then conducted across correlation values ( $Z$-transformed) acquired for pairs of independent components. Betweennetwork connectivity differences between timepoints were investigated using non-parametric paired testing, with a statistical significance threshold set at $p<0.05$, FWE-corrected.

\section{RESULTS}

\section{Clinical and Conventional MRI Data}

Demographic and clinical characteristics of patients at Tst are reported in Table 1 . Thirty patients were scanned at $\mathrm{T} 6 \mathrm{~m}$ and included in the analyses. Eleven were treatment-naïve, 17 switched from a previous first-line DMT, and 2 switched from a second-line DMT. The switch from a first-line DMT was due to inefficacy, while the switch from a second-line DMT (natalizumab) was due to progressive multifocal leukoencephalopathy risk. Three patients dropped out after $16 \mathrm{~m}$ because of their unavailability to follow the protocol time schedule. Therefore, 27 patients were scanned at $\mathrm{T} 12 \mathrm{~m}$.

Scores obtained on the clinical/neuropsychological assessment and radiological features at Tst, $\mathrm{T} 6 \mathrm{~m}$, and $\mathrm{T} 12 \mathrm{~m}$ are reported in Table 2.

Table 1 Demographic and clinical characteristics of our cohort of MS patients $(N=30)$ at therapy start (Tst)

\begin{tabular}{ll}
\hline MS patients $(\boldsymbol{N}=\mathbf{3 0})$ & \\
\hline Demographic/clinical features & $41.9 \pm 10.2$ \\
Age & $25 / 5$ \\
Female/male, $n$ & $11.0 \pm 8.2$ \\
Disease duration, years & $4(13)$ \\
Pts. with relapse in previous year, $n(\%)$ & $80[68-360]$ \\
Time since last relapse, days [range] & $11(37)$ \\
Treatment naïe, $n(\%)$ & $17 / 2$ \\
First/second-line therapy, $n$ &
\end{tabular}

Values are reported as the mean \pm standard deviation

${ }^{\S}$ In patients with a relapse in previous year 
Table 2 Scores obtained in the clinical/neuropsychological assessment and radiological features at therapy start (Tst), after 6 months $(\mathrm{T} 6 \mathrm{~m})$, and after 12 months (T12m) of DMF treatment and statistical comparison results (Wilcoxon signed-rank test with threshold of $p<0.05$ )

\begin{tabular}{|c|c|c|c|c|c|}
\hline MS patients & Tst $(N=30)$ & T6m $(N=30)$ & T12m $(N=27)$ & $p^{*}$ & $p^{* *}$ \\
\hline \multicolumn{6}{|l|}{ Clinical/neuropsychological scores } \\
\hline EDSS score [median (range)] & $1.5[0.0-4.0]$ & $1.5[0.0-5.5]$ & $1.5[0.0-5.5]$ & $\mathrm{ns}$ & ns \\
\hline Pts. with relapse under DMF, $n(\%)^{\dagger}$ & - & $1(3)$ & $1(4)$ & - & - \\
\hline 9-HPT dominant hand, $s$ & $20.0 \pm 3.4$ & $20.0 \pm 3.0$ & $19.5 \pm 2.9$ & $\mathrm{~ns}$ & ns \\
\hline 9-HPT non-dominant hand, $s$ & $21.4 \pm 4.8$ & $21.6 \pm 4.0$ & $21.0 \pm 3.6$ & ns & ns \\
\hline 25-FWT, s & $6.4 \pm 1.4$ & $6.5 \pm 1.7$ & $6.0 \pm 1.4$ & ns & 0.005 \\
\hline PASAT $3, s$ & $39.8 \pm 15.2$ & $42.0 \pm 13.4$ & $43.5 \pm 12.0$ & ns & 0.022 \\
\hline PASAT 2, s & $32.5 \pm 13.1$ & $33.4 \pm 10.7$ & $33.4 \pm 12.8$ & ns & ns \\
\hline SDMT & $44.2 \pm 12.3$ & $44.1 \pm 13.1$ & $43.0 \pm 13.6$ & ns & ns \\
\hline \multicolumn{6}{|l|}{ Radiological features } \\
\hline Brain volume $\left(\mathrm{cm}^{3}\right)$ & $1,407 \pm 259$ & - & - & - & - \\
\hline Gray matter volume $\left(\mathrm{cm}^{3}\right)$ & $743 \pm 59$ & - & - & - & - \\
\hline T2-lesion volume $\left(\mathrm{cm}^{3}\right)$ & $8.997 \pm 10.700$ & $9.215 \pm 10.948$ & $9.770 \pm 11.332$ & ns & ns \\
\hline Percentage brain volume change $(\%)^{\dagger}$ & - & $\begin{array}{l}-0.12 \\
\quad[-1.83-1.88]\end{array}$ & $\begin{array}{l}-0.24 \\
\quad[-2.72-1.77]\end{array}$ & - & - \\
\hline $\begin{array}{l}\text { Pts with new/enlarging lesions under DMF, } \\
n(\%)^{\dagger}\end{array}$ & - & $6(20)$ & $6(22)$ & - & - \\
\hline Pts with gadolinium-positive lesion, $n$ (\%) & $3(10)$ & $1(3)^{\dagger}$ & $1(4)^{\dagger}$ & - & - \\
\hline
\end{tabular}

Values are reported as the mean \pm standard deviation or median $[\mathrm{min}-\mathrm{max}]$

$s$ seconds, $n s$ not statistically significant

9HPT 9-Hole Peg Test, 25FWT, 25-Feet Walk Test, PASAT Paced Auditory Serial Addition Test 3 and 2 s, SDMT Symbol Digit Modalities Test

*Differences between Tst and T6m

**Differences between Tst and T12m

${ }^{\dagger}$ Respect to baseline

After 6 months of DMF $(N=30)$, two patients showed disability worsening and six showed MRI activity (new/enlarged T2 or gadoliniumenhancing lesions). Of these six, one dropped out of the study. In the following 6 months, only one other patient showed a new T2 lesion and another one showed disability progression.

With respect to baseline, SIENA showed a median PBVC of $-0.12[-1.83-1.88]$ after 6 months and of $-0.24[-2.72-1.77]$ after 12 months of DMF.
No significant longitudinal changes were found with respect to clinical/neuropsychological or radiological scores after 6 months of treatment. After 12 months, there was significant longitudinal improvement in PASAT $3 \mathrm{~s}$ and 25-FWT scores (PASAT 3 s: $p=0.022 ; 25-\mathrm{FWT}: p=0.005$ ).

\section{Functional Connectivity}

No significant longitudinal changes in primary motor cortex FC were found after either 6 or 
12 months of therapy $(p<0.05, \quad$ FWEcorrected).

ICA yielded 30 independent components. Of these, we identified ten components that showed the highest spatial correlation coefficients with RSN templates: the default mode $(r=0.76)$, dorsal attention $(r=0.51)$, left and right frontoparietal $(r=0.64$ and $r=0.61$, respectively), executive control $(r=0.62)$, visual $(r=0.69)$, cerebellar $(r=0.49)$, orbitofrontal $(r=0.39)$, auditory $(r=0.62)$, and sensorimotor $(r=0.59)$ networks.

No significant longitudinal FC changes in within- or between-network FC were found after either 6 or 12 months of therapy $(p<0.05$, FWE-corrected).

\section{DISCUSSION}

In the present study, we investigated possible FC changes after an initial 6-month treatment with DMF and after a longer follow-up period of 12 months. When applying the same methodological approach as in our previous studies $[2,3]$, we did not find any significant FC changes after DMF treatment. After 12 months of DMF, we observed relatively modest brain volume loss and a significant improvement in PASAT $3 \mathrm{~s}$ and 25-FWT scores. One possible explanation for the absence of any significant FC changes after therapy is the low degree of baseline inflammation at baseline. In fact, gadolinium-enhancing lesions were present in only three patients at therapy start, whereas in our previous studies on fingolimod effects [2,3], 15 out of 30 patients showed gadolinium-enhancing lesions. Intrinsic neural activity is generally regulated by excitatory/inhibitory balance, which is closely related to the levels of glutamate and gamma-aminobutyric acid [17]. In the acute MS stage, a large quantity of glutamate is produced by activated leukocytes, macrophages, and microglia [18], as also demonstrated by MR spectroscopy [19]. Due to glutamate excitatory action, it is reasonable to assume that acute inflammation in MS may be linked to increased neural activity [20, 21], which may be reduced by anti-inflammatory drugs. The hypothesis that the effect on FC might be related to inflammation prior to therapy initiation is supported by evidence in the literature: the effect of DMTs on brain plasticity is more pronounced in patients who have a greater degree of central nervous system inflammation [22].

It is also possible that DMF needs more time to modulate FC, especially in the presence of a relatively low number of patients with active inflammation. A recent 2-year study on fingolimod effects on FC [23] showed no DMT effect until the second year. The authors ascribed this delayed effect to the inclusion of patients without active inflammation at therapy start.

Lastly, we cannot exclude the possibility that the lack of FC changes after therapy may reflect a positive phenomenon, i.e., stability, as conventional MRI and clinical data suggest. As a matter of fact, after 12 months of therapy we observed modest brain volume loss $(-0.24 \%)$, which was inferior to the reference annual rate of brain volume loss in MS patients (from - 0.4 to $-1.0 \%)[24,25]$. This result is consistent with a previous study on DMF-treated MS patients, which suggested a treatment effect on GM atrophy after 1 year of therapy [26].

We also observed an improvement in PASAT $3 \mathrm{~s}$ and 25-FWT scores. PASAT improvement may be due to a practice or learning effect; this seems to be supported by the absence of improvement in SDMT, which is less prone to such effects [27]. Regarding walking speed, the observed improvement in 25-FWT scores (6.25\%) may not be clinically meaningful (an improvement $\geq 20 \%$ is generally considered as such); however, it is surely indicative of clinical stability [28].

Future studies with larger sample sizes and longer follow-up observations are needed to confirm the present findings and draw firm conclusions about the effects of DMF therapy on neural functioning in MS patients.

\section{Study Limitations}

One of the main limitations of the present study was the relatively small sample size $(N=30)$. However, we were previously able to detect 
significant FC changes after 6 months of fingolimod treatment in 30 RR-MS patients who were scanned using the same scanner and sequences as those described in the present study and who had similar clinical characteristics to those of the present patient cohort $[2,3]$. Furthermore, it has been demonstrated that even a smaller number of patients $(N=24)$ can show significant functional changes after oral DMT (interferon beta) [22].

Another main limitation was the lack of a control group of healthy subjects or a group of untreated patients. Although comparison with healthy subjects would have allowed us to characterize any FC changes as patient specific, previous studies have shown that MS patients and healthy subjects have different baseline connectivity in most RSNs [29]. The longitudinal study design was also meant to compensate for the lack of an untreated MS patient group, which would be unethical.

Lastly, the anti-inflammatory effects of DMF were evaluated only by means of conventional MRI. The use of other techniques, such as positron emission tomography, would have assessed the inflammation of brain tissue outside the lesion areas [30]. Furthermore, it is known that even when the blood-brain barrier is intact, infiltrates of activated microglia and macrophages, an expression of chronic inflammation, have been demonstrated in patients with MS and have been related to clinical disability [31]. Thus, we cannot exclude the presence of neuroinflammation in our patient sample, even in the absence of gadolinium-enhancing white matter lesions.

\section{CONCLUSION}

We did not observe FC changes after 6 months of DMF therapy, unlike our previous findings in fingolimod-treated patients [2, 3]. Furthermore, we did not observe FC changes after a longer follow-up period of 12 months. This could be explained by the low degree of baseline inflammation in our patients, suggesting that more time may be required to observe FC changes. An alternative explanation, also supported by conventional MRI findings and clinical improvement, is that the absence of FC changes reflects the absence of (further) impairment to the functional network, which can be regarded as preservation of brain function.

\section{ACKNOWLEDGEMENTS}

We thank the participants of the study.

Funding. No funding or sponsorship was received for this study or publication of this article. The Rapid Service Fee was funded by the authors.

Authorship. All named authors meet the International Committee of Medical Journal Editors (ICMJE) criteria for authorship for this article, take responsibility for the integrity of the work as a whole, and have given their approval for this version to be published.

Author Contributions. Claudia Piervincenzi contributed to method definition, data analysis, and manuscript editing. Nikolaos Petsas contributed to data analysis and manuscript editing. Emilia Sbardella and Marta Altieri contributed to study design and recruitment, data interpretation and manuscript editing. Carlo Pozzilli and Antonio Ianniello contributed to data interpretation and manuscript editing. Patrizia Pantano supervised the study and contributed to data interpretation and manuscript editing.

Disclosures. Nikolaos Petsas received speaker fees from Biogen Idec and mission support from Merck Serono, Genzyme, and Novartis. Carlo Pozzilli received consulting and lecture fees from Sanofi-Aventis, Biogen Idec, Bayer Schering, Merck Serono, and Novartis. He also received research funding from Novartis, Sanofi-Aventis, Merck Serono, and Bayer Schering. Patrizia Pantano received travel funding from Novartis, Genzyme, and Bracco and speaker honoraria from Biogen Idec. Claudia Piervincenzi, Emilia Sbardella, Marta Altieri and Antonio Ianniello declare that the research was 
conducted in the absence of any commercial or financial relationships that could be construed as a potential conflict of interest.

Compliance with Ethics Guidelines. This study was performed in accordance with the ethical code of the ethics committee of Azienda Policlinico Umberto I, Sapienza University of Rome (Rif. 2984/12.12.2013), and the Declaration of Helsinki. After approval from the ethics committee, written informed consent was obtained from all subjects.

Data Availability. The datasets generated during and/or analyzed during the current study are not publicly available because of patient confidentiality and participant privacy restrictions. Requests to access the datasets should be directed to petsas@gmail.com.

Open Access. This article is licensed under a Creative Commons Attribution-NonCommercial 4.0 International License, which permits any non-commercial use, sharing, adaptation, distribution and reproduction in any medium or format, as long as you give appropriate credit to the original author(s) and the source, provide a link to the Creative Commons licence, and indicate if changes were made. The images or other third party material in this article are included in the article's Creative Commons licence, unless indicated otherwise in a credit line to the material. If material is not included in the article's Creative Commons licence and your intended use is not permitted by statutory regulation or exceeds the permitted use, you will need to obtain permission directly from the copyright holder. To view a copy of this licence, visit http:// creativecommons.org/licenses/by-nc/4.0/.

\section{REFERENCES}

1. Rocca MA, Preziosa P, Filippi M. Application of advanced MRI techniques to monitor pharmacologic and rehabilitative treatment in multiple sclerosis: current status and future perspectives. Expert Rev Neurother. 2019;19:835-66.
2. Petsas N, De Giglio L, González-Quintanilla V, Giuliani M, De Angelis F, Tona F, et al. Functional connectivity changes after initial treatment with fingolimod in multiple sclerosis. Front Neurol. 2019;10:153.

3. Piervincenzi C, Petsas N, De Giglio L, Carmellini M, Giannì $\mathrm{C}$, Tommasin $\mathrm{S}$, et al. Increased withinnetwork functional connectivity may predict NEDA status in fingolimod-treated MS patients. Front Neurol. 2021;12:632917.

4. Giovannoni G, Turner B, Gnanapavan S, Offiah C, Schmierer K, Marta M. Is it time to target no evident disease activity (NEDA) in multiple sclerosis? Mult Scler Relat Disord. 2015;4:329-33.

5. Gold R, Kappos L, Arnold DL, Bar-Or A, Giovannoni G, Selmaj K, et al. Placebo-controlled phase 3 study of oral BG-12 for relapsing multiple sclerosis. N Engl J Med. 2012;367:1098-107.

6. Fox RJ, Miller DH, Phillips JT, Hutchinson M, Havrdova E, Kita M, et al. Placebo-controlled phase 3 study of oral BG-12 or glatiramer in multiple sclerosis. N Engl J Med. 2012;367:1087-97.

7. Linker RA, Gold R. Dimethyl fumarate for treatment of multiple sclerosis: mechanism of action, effectiveness, and side effects. Curr Neurol Neurosci Rep. 2013;13:394.

8. Polman $\mathrm{CH}$, Reingold SC, Banwell B, Clanet $\mathrm{M}$, Cohen JA, Filippi M, et al. Diagnostic criteria for multiple sclerosis: 2010 revisions to the McDonald criteria. Ann Neurol. 2011;69:292-302.

9. Río J, Nos C, Tintoré M, Téllez N, Galán I, Pelayo R, et al. Defining the response to interferon-beta in relapsing-remitting multiple sclerosis patients. Ann Neurol. 2006;59:344-52.

10. Beckmann CF, DeLuca M, Devlin JT, Smith SM. Investigations into resting-state connectivity using independent component analysis. Philos Trans R Soc Lond B Biol Sci. 2005;360:1001-13.

11. Filippini N, MacIntosh BJ, Hough MG, Goodwin GM, Frisoni GB, Smith SM, et al. Distinct patterns of brain activity in young carriers of the APOE-epsilon4 allele. Proc Natl Acad Sci U S A. 2009;106: 7209-14.

12. Smith SM, Fox PT, Miller KL, Glahn DC, Fox PM, Mackay CE, et al. Correspondence of the brain's functional architecture during activation and rest. Proc Natl Acad Sci U S A. 2009;106:13040-5.

13. Yeo BTT, Krienen FM, Sepulcre J, Sabuncu MR, Lashkari D, Hollinshead M, et al. The organization of the human cerebral cortex estimated by intrinsic 
functional connectivity. J Neurophysiol. 2011;106: 1125-65.

14. Worsley KJ. Statistical analysis of activation images [Internet]. Oxford University Press; 2000. https:// oxford.universitypressscholarship.com/view/10. 1093/acprof:oso/9780192630711.001.0001/acprof9780192630711-chapter-14.

15. Nichols TE, Holmes AP. Nonparametric permutation tests for functional neuroimaging: a primer with examples. Hum Brain Mapp. 2002;15:1-25.

16. Smith S, Nichols $\mathrm{T}$. Threshold-free cluster enhancement: addressing problems of smoothing, threshold dependence and localisation in cluster inference. Neuroimage. 2009;44:83-98.

17. Duncan NW, Wiebking C, Northoff G. Associations of regional GABA and glutamate with intrinsic and extrinsic neural activity in humans-a review of multimodal imaging studies. Neurosci Biobehav Rev. 2014;47:36-52.

18. Kelley AE, Andrzejewski ME, Baldwin AE, Hernandez PJ, Pratt WE. Glutamate-mediated plasticity in corticostriatal networks: role in adaptive motor learning. Ann N Y Acad Sci. 2003;1003:159-68.

19. Srinivasan R, Sailasuta N, Hurd R, Nelson S, Pelletier D. Evidence of elevated glutamate in multiple sclerosis using magnetic resonance spectroscopy at 3 T. Brain. 2005;128:1016-25.

20. Colasanti A, Guo Q, Giannetti P, Wall MB, Newbould RD, Bishop C, et al. Hippocampal neuroinflammation, functional connectivity, and depressive symptoms in multiple sclerosis. Biol Psychiatry. 2016;80:62-72.

21. Stampanoni Bassi M, Gilio L, Buttari F, Maffei P, Marfia GA, Restivo DA, et al. Remodeling functional connectivity in multiple sclerosis: a challenging therapeutic approach. Front Neurosci. 2017;11:710.

22. Tomassini V, d'Ambrosio A, Petsas N, Wise RG, Sbardella E, Allen M, et al. The effect of inflammation and its reduction on brain plasticity in multiple sclerosis: MRI evidence: inflammation and brain plasticity in MS. Hum Brain Mapp. 2016;37: 2431-45.

23. Bhattacharyya PK, Lowe MJ, Sakaie KE, Li H, Lin J, Fox RJ. Changes in structural and functional connectivity during two years of fingolimod therapy for multiple sclerosis. Magn Reson Imaging. 2020;74:113-20.

24. Stefano ND, Giorgio A, Battaglini M, Rovaris $M$, Sormani MP, Barkhof F, et al. Assessing brain atrophy rates in a large population of untreated multiple sclerosis subtypes. Neurology. 2010;74:1868-76.

25. De Stefano N, Airas L, Grigoriadis N, Mattle HP, O'Riordan J, Oreja-Guevara C, et al. Clinical relevance of brain volume measures in multiple sclerosis. CNS Drugs. 2014;28:147-56.

26. Dupuy SL, Tauhid S, Hurwitz S, Chu R, Yousuf F, Bakshi R. The effect of dimethyl fumarate on cerebral gray matter atrophy in multiple sclerosis. Neurol Ther. 2016;5:215-29.

27. Amato MP, Portaccio E, Goretti B, Zipoli V, Iudice A, Della Pina D, et al. Relevance of cognitive deterioration in early relapsing-remitting MS: a 3-year follow-up study. Mult Scler. 2010;16:1474-82.

28. Kaufman M, Moyer D, Norton J. The significant change for the Timed 25-foot Walk in the multiple sclerosis functional composite. Mult Scler. 2000;6: 286-90.

29. Rocca MA, Valsasina P, Martinelli V, Misci P, Falini A, Comi G, et al. Large-scale neuronal network dysfunction in relapsing-remitting multiple sclerosis. Neurology. 2012;79:1449-57.

30. Poutiainen P, Jaronen M, Quintana FJ, Brownell A-L. Precision medicine in multiple sclerosis: future of PET imaging of inflammation and reactive astrocytes. Front Mol Neurosci. 2016;9:85.

31. Herranz E, Giannì C, Louapre C, Treaba CA, Govindarajan ST, Ouellette R, et al. Neuroinflammatory component of gray matter pathology in multiple sclerosis. Ann Neurol. 2016;80:776-90. 